\title{
Viral Musicality in Harmony with Nature
}

\author{
Theaux $\mathrm{C}^{1}$, Scolaro $\mathrm{LA}^{1,2}$ and Carlucci $\mathrm{MJ}^{1,2 *}$
}

${ }^{1}$ Virology Laboratory QB19, Department of Biological Chemistry, Faculty of Exact and Natural Sciences, University of Buenos Aires, Buenos Aires, Argentina; ${ }^{2}$ Institute of Biological Chemistry, Faculty of Exact and Natural Sciences (IQUIBICEN), UBA-CONICET, Buenos Aires, Argentina

\begin{abstract}
In this manuscript we describe a biological system that deals with herpes simplex virus in the context of an in vitro infection in the presence of carrageenan, from a novel point of view by comparing our system to the Ligeti's symphonic poem of 100 metronomes. Metronomes (from ancient Greek $\mu \varepsilon ́ \tau \rho o v$-métron, "measure" and vé $\mu \omega$-némo, "I manage", "I lead"), as a measuring instrument of "tempo" links us with the viral replication cycle to define, through sound, a metaphorical construction of life (as vibration and movement, impermanence and constant change). The changes detected in viral populations, in time and space, in a similar way to the artistically experienced in Ligeti's symphonic poem enable us a trans disciplinary view, creating networks of cooperation that favor the renewal of the conceptual bases of biology enriching our perception and understanding of the biology and evolution of viruses.
\end{abstract}

Keywords: Virus; Evolution; Epigenetic; Horizontal gene transfer; Herpes simplex virus; Carrageenans; Metronome model

\section{INTRODUCTION}

Experimental models proposed by the scientific community usually are symbolic representations that intent to describe the main features or dimensions of the phenomena they represent as accurately as possible. Useful models can be formalized into mathematical equations or into a computational algorithm [1]. As such, they are suitable for decomposing complex phenomena into simpler and more easily understood representations. However, the organizational complexity of biological systems challenges their modeling and mathematical analysis leading to the dismissal of many biological significative variables in order to fit the model [2]. The main problem arises when researchers assume that the motivation of a finding is based exclusively on the specific model they defend. Such assumptions ignore the complexity of nature and its richness and thus lose sight of the interpretations and alternative models. In addition, phenomena that are outside the scope of the model will tend to be excluded from all consideration or misinterpreted. In this line, many variables that cannot be represented by variations of concentration or quantity over time are usually omitted in rule-based models in biology [3]. In this way, most of the models that deal with the study of viral populations characterized by the appearance of genetic changes are those subjected to extreme environmental pressures with mutagenic drugs, but what happens if we use instead non-mutagenic compounds such as polysaccharides known as Carrageenans (CGNs) and we still detect genetic changes in the population? These results that come out of the expected motivate a new point of view of the environmental effect in the viral populations enriching the interpretations of the experimental model.

A growing trend is the incorporation of art in everyday life. In this line, "Fluxus", a controversial and provocative artistic movement of the twentieth century, denounces the notion of art as a product for a select audience and proposes a way of life impregnated with a superb freedom to think, express and choose. In the same way we can think science. However, certain restrains or rules should be considered in order to avoid pure intuition in the construction of the model, otherwise everything we perceive tends to tell us that our assumptions and beliefs are correct [4].

In recent years, the image of the world presented to us by physicists has undergone a radical change and implications so vast as to touch the very foundations of science. Traditional descriptions were based largely on Greek philosophical concepts and the universe was described as atomistic, divisible, static and non-relativistic. These descriptions now need the supplement of models that recognize a holistic, indivisible, interconnected, dynamic and relativistic reality, which is not only inseparable from the consciousness of the observer, but also a function of it [4]. In this respect, music, a branch of art in which sounds, and silences are constrained by

Correspondence to: Carlucci MJ, Virology Laboratory QB19, Department of Biological Chemistry, Faculty of Exact and Natural Sciences, University of Buenos Aires, Buenos Aires, Argentina, Tel: +54-11-4576-3334; E-mail: majoc@qb.fcen.uba.ar

Received: April 23, 2019, Accepted: May 13, 2019, Published: May 20, 2019

Citation: Theaux C, Scolaro LA, Carlucci MJ (2019) Viral musicality in harmony with nature. Biol Med (Aligarh) 11:461. doi: 10.35248/0974-8369.19.11.461

Copyright: ( 2019 Theaux C, et al. This is an open-access article distributed under the terms of the Creative Commons Attribution License, which permits unrestricted use, distribution, and reproduction in any medium, provided the original author and source are credited. 
time, makes a valuable complement of mathematical models in biology. Viruses acting as population, are difficult to fit with the basic concepts of evolutionary dynamics of Darwinian natural selection and neo-Darwinism because there are other forces leading to hereditary changes, such as symbiogenesis, hybridogenesis, and horizontal gene transfer, which collectively expand our horizons to a diversity of possibilities [5,6]. In this paper we present a model of viral evolution of herpes simplex virus subjected to a selective pressure exerted by Carrageenan (CGNs), a natural antiviral compound, based on Ligeti's symphonic poem of 100 metronomes.

\section{SYMPHONIC POEM OF 100 METRONOMES AND THE EXPERIMENTAL MODEL}

Ligeti's ideas and his symphonic poem constitute an example of how to interpret the flow in life and in art, as characterized by the Fluxus art movement [7]. Despite the seemingly random organicity in the Symphonic Poem, this is a work completely embedded in a process of perpetual continuum in which, however, there are elements of constant discontinuity, which make it a work of quantum character, with the singularity of constituting a unique and complex process.

In this work, the periodicity in the sound is perceived as a point in the creative process of Nature, it also reflects the fascination of the composer by automated mechanisms, in this case a phase shift of 100 metronomes where the only human role is to trigger the phenomenon: Turn them on. The shape of the sound mass that was generated could be outlined as U-C-U' (Uniformity, Chaos, Uniformity):

$\mathrm{U}$ : Uniformity: continuous sound mass with all the metronomes active at the same time, the great sound mass hides a rhythmic randomness.

C: Chaos: continuous changes when the metronomes started to stop; sound variations are heard because they do not stop simultaneously.

U': Uniformity: back to uniformity when only one or a few metronomes remain with continuous rhythm; easily perceived and heard so clear that they can define an order. "This is music that gives the impression that could flow continuously, as if it had no beginning or end; what we hear is actually a section of something that has begun and will continue ringing eternally forever. The formal characteristic of this music is that it feels static. Music seems to be quiet but that is just an illusion: in this stillness within this static quality, there are gradual changes" [8].

This artwork can be taken as a metaphor for life processes or social time. Time course acting on biological systems in nature. Each metronome may represent a virus, a microorganism or a more complex organism (human) and together they are mechanically 'living' in their own way.

Masses of sound and families of clicks that sound synchronously are waiting a moment to return to chaos. And along this road, the last beat of the last metronome is disturbing, because instinctively we associate movement with life.

By definition, a random universe evolves by chance or accident and its fate would be therefore entirely unpredictable. However, everything that seems random may be not: it can also be chaotic. Random systems and chaotic systems are very similar from the outside, however random systems act from the accident, while chaotic, although seemingly random, actually rely on an underlying organization [9]. The terms randomness and chaos together with order, can be used to describe the organizational complexity of a system.

In our experimental model successive passages of HSV in cell cultures were used with the addition of small increasing doses of CGN and its adaptations through its phenotypic and genotypic modifications were analyzed. The CGNs, sulphated galactans derived from red seaweed, with chemical structures similar to cellular receptor heparin sulfate were identified as potent and selective inhibitors of herpes simplex virus. Their mechanism of action mainly affects viral adsorption stage, interacting with the surface glycoproteins. In Figure 1, the changes of viral titers for each passage with or without CGNs are shown, using virus passages in the presence of Acyclovir (ACV) as control. The appearance of resistant variants was seen in a few passages for ACV. HSV-1 and HSV-2 variants, exhibited similar biological characteristics: low resistance to CGNs, moderate resistance to ACV and decreased virulence with differentiated activation of pro-inflammatory cytokines with respect to the parental strain. From passage 11 onwards, changes were detected at the level of the cytopathic effect of the virus. In the early passages cell rounding was observed, first as small foci that eventually spread over the entire cell monolayer. Multinucleated cells forming a syncytium due to the fusion of adjacent infected cells were also observed. Also, in this passage, a marked change was detected in the size of the viral plaques, coexisting small viral plaques $(1 \mathrm{~mm})$ (similar to the parental strain) and large viral plaques of $1.5-2 \mathrm{~mm}$, until passage 16 , from which homogeneity of large plaques as syncytium was maintained [10]. When viral titer decreases in 1.5-2 log (Figure 1) a viral amplification was performed using minimal amount of CGN.

Surprisingly, a preliminary study of structural and nonstructural genes of the variants by Next Generation Sequencing (NGS) revealed that, in addition to the glycoproteins, the variants have altered genes that would not be related to the target of action of CGN as thymidine kinase and viral DNA polymerase $[10,11]$. These genes are responsible for pathogenicity and virulence.

As shown in the Figure 2, randomness and order represent extreme poles of the organizational structure in which the chaos occupies the central position.

\section{THE COMPLEX AND AMAZING INTERPR- ETATION OF NATURE}

\section{Chaotic systems, Newtonian and quantum physics}

Random systems grow with uncertainty and, therefore, cannot

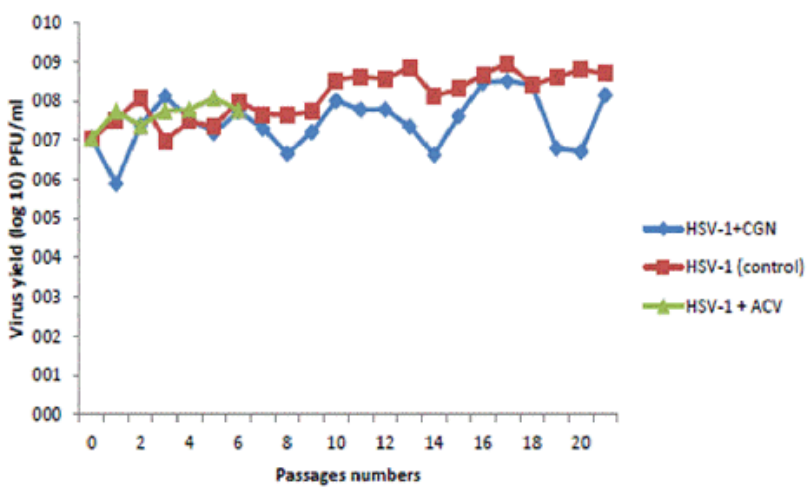

Figure 1: Passages of HSV-1 in the presence and absence of CGN. 


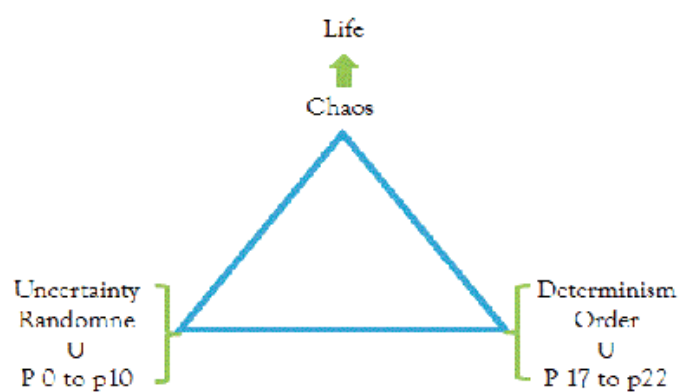

Figure 2: Organizational Structure.

sustain life because they lack the necessary organization to provide a regulated and integrated physiology. On the other hand, life cannot arise out of a rigid crystal system because it does not offer the dynamism required by living organisms, life requires a suitable system, and finds it in the fertile predictability of dynamic and controllable chaos. The capacity to predict the fate of a system is based on the nature of its organization. When we know the patterns that determine the highly ordered systems, we can accurately predict their past and future conditions. Instead, in random systems, the inherited irregular behavior makes it difficult, or even impossible, to hit prediction. The organization of a system, and therefore the ability to predict its fate, are established in the physics mechanisms governing these operations. Systems employing Newtonian physics include determinism and order, while quantum mechanics added uncertainty to the equation. By contrast, chaotic systems are characterized by order and disorder and therefore are configured by Newtonian and quantum physics. The fate of living systems receives the simultaneous influence of the characteristics of both determinism and uncertainty. Heisenberg's theory about uncertainty is applicable to any pair of conjugate variables, such as position and velocity, time and energy or rotation angle and angular moment. The theory implies that the measurement of a variable derives in the alteration of its conjugate pair, what follows that it is never possible to predict precisely two variables at the same time. Keeping in mind the thought of Rob Shaw, "Chaos is the creator of information", we wanted to analyze a process of population variability influenced by the environment based on the concept of sensitivity analyzed by Edward Lorenz for complex dynamic systems.

The concept of sensitivity emphasizes that extremely small differences in initial conditions can generate significant consequences that are then perceived as random changes. That is to say: insignificant differences in the input quickly turn into huge differences in the output, a phenomenon that was called "sensitive dependence on initial conditions" [12]. Thus, the use of high concentrations of CGNs decreases most of the viral population due to its strong antiviral effect selecting the resistant virus, however, very low doses of CGNs produce large population changes.

Bearing in mind that the laws of quantum mechanics do not deny the results of Newtonian mathematics, the difference between both types of physics lies in the fact that quantum mechanics is applied especially at the atomic and molecular levels, manifesting itself as vibration, waveform, as electromagnetic fields itself or even sounds while the Newtonian laws are applied specially to higher levels of organization, such as organic systems, people or groups of people.

A viral disease can be detected at a higher level when the symptoms are seen or felt, however the process that caused the disease began at the molecular level, inside the affected cells. Hence, the need for the biology of viruses to integrate both quantum and Newtonian mechanics.

\section{Biomimicry system and viral population}

In our studies we subjected a viral population to increasing concentrations of CGN, which functioned as an environmental selective agent, in order to determine the resistance of the resulting population to that agent that we expected it would be greater than that observed for parental virus or for passaged virus in the absence of CGN. However, we found little resistance in the viral population to CGN. More than $96 \%$ of the population exhibited a common nucleotidic change at the level of $\mathrm{gB}$, resulting in an attenuated population [12]. We know that changing the concentrations of the compounds their mechanisms of action will also change. Thus, CGNs in high concentrations proved to be antivirals but at low levels they can act as modulators of cellular and viral signals. After the initial stage of infection where the virus meets CGNs, comparable to Ligeti's uniformity stage when all metronomes went off, the second stage of the process may be compared to the chaos stage in Ligeti's symphony. In this stage a highly variable viral population struggles to overcome antiviral action. During the successive passages, chaos returns to uniformity due to the appearance of viruses that may replicate in the presence of CGNs. At the molecular level, we can consider that CGNs has vibrational resonances compatible with HSV virions that favor the stabilization-adaptation between the polymer and the viral glycoproteins. CGNs bio-mimicked with cellular receptors, targets for glycoproteins interaction $[13,14]$. The binding of HSV to the cell involves glycoproteins $\mathrm{gC}, \mathrm{gB}$ and $\mathrm{gD}$ whereas $\mathrm{gB}, \mathrm{gH}$ and $\mathrm{gL}$ participate in the fusion process between viral and cell membranes [15]. Apparent random interactions between CGNs and glycoproteins during the chaos stage led to the appearance of syncitial variants from passage 11 onwards [10].

The complexity of the model augments if we consider that the interactions between CGNs and glycoproteins may be transmitted towards the inside of the virion, via proteins of the viral tegument that respond by changing their folding through the interaction with water molecules [16-18]. These changes in protein folding may reach viral DNA ultimately. In $\mathrm{HSV}$, the mature virion contains the viral DNA in the form of a torus $[19,20]$. In some herpes virions, the torus appears to be suspended by a proteinaceous spindle consisting of fibrils embedded in the underside of the capsid and passing through the hole of the torus and connecting with tegument. These proteins could have similar activity to the regulatory proteins that surround our DNA; these are the ones that would allow the epigenetic effects to define which genes to activate [21]. In consequence, the multiplicity of factors involved in virus binding/penetration adds variability to the model and augments the level of chaos of this stage of the system [22-26].

\section{CONCLUSION}

Everything in Nature is in process, it is simply the flow, the course of the journey of life, adapting to changes in the environment. Every process is dynamic, a river of rhythm and form. We know that there is a continuous oscillation between order and chaos, fundamental to the very nature of the process. To unveil the pattern, to become aware of it, many times we need to allow the process to submerge in chaos, so that a new order, a new and more appropriate pattern can come to light, the way it happens with Ligeti's symphony of metronomes. These periods of chaos are the transition points, without them, the process will not evolve towards the new and 
"healthier" pattern. At these transition points, the coherence of the established pattern is momentarily lost so that a new pattern can be found, giving continuity to the process.

Creating favorable conditions, aspiring to enable and allow, without imposing, the new is born alone. In our model, we used low concentrations of CGNs in each passage facilitating adaptation or adjustment to the viral population. In a quantum look the system constituted by a block finite and separate of CGNs-virus-cell has disappeared. Discovering that these components change their forms (folding of the polymer of CGNs, viral and cellular protein folding) and their properties in relation to others (CGNs-cells, CGNs-virus, virus-cell) interacting among them. Under this sight, the world seems to consist of "relationships" instead of "things". And what we think of as "things" are in fact intermediate stages in a network of interactions and relationships that are constantly changing. Therefore, systems (and every living organism is a system), are not reducible or predictable, everything depends on the unique relationships that are established and disappear in a flow and ebb, moving from chaos to order and vice versa. Music is incoherent noise if it is heard in fragments, but by apprehending the whole, noise transforms into music. This is the case of the work of Ligeti, it is noise if it is fragmented with respect to the rest of the sounds of the whole. In our case with the cellular vibratory sound, the chains of the CGNs that change their vibratory intensities depending on their concentration and interactions with viral proteins creating a great melody, that reflects the success of the adaptive process.

A way of thinking based on polarity between order and chaos causes a stagnation of the life process. Finding balance between both stages is much more complex but it seems to be the basis of creativity and development. Both polarities are in their absolute power and meaning, at the same level, so that tension and energy increase. In this way, to evolve we must rise above duality, unifying and taking the best of each end, bringing the polarities closer to create the third force that is balance, a flow and ebb between them. Viral evolution is the result of the system that tends to maintain the balance in a flexible and fluid manner; this "fluxus" keeps the virus in tune with itself and the environment through moments of noise and melody.

\section{FUNDING}

Research in the author's laboratory was supported by the National Council of Scientific and Technological Research (CONICET). MJC and LAS are members of the Research Career from CONICET.

\section{REFERENCES}

1. Berro J. Essentially, all models are wrong, but some are useful-a crossdisciplinary agenda for building useful models in cell biology and biophysics. Biophys Rev. 2018;10(6):1637-1647.

2. Gadomski A, Bier M, Siódmiak J. Spatiotemporal models in biology and the health sciences. Biosystems. 2019;179:15-16.

3. Bustos Á, Fuenzalida I, Santibáñez R, Pérez-Acle T, Martin AJM. Rule-based models and applications in biology. Methods Mol Biol. 2018;1819:3-32.

4. Walsh R, Vaughan F. Mas alla del Ego, Editorial Kairos. Barcelona, Spain. 1982.

5. Mateu CG, Artuso MC, Pujol CA, Linero FN, Scolaro LA, Carlucci MJ. In vitro isolation of variant of herpes simplex virus attenuated with altered thymidine kinase and DNA polymerase genes using carrageenans as selection agents. Symbiosis. 2017;72(1):23-30.
6. Villarreal LP, Ryan F. Viruses in host evolution: general principles and future extrapolations. Curr Top Virol. 2011;9:79-90.

7. Jenkins J, Rothfuss J. Edit, In the spirit of Fluxux. Walker art center. 1993.

8. Ligeti G. "LIGETI in conversation" with Peter Varnai, Josec Häusler, Claude Samuel and himself. Eulenburg books. Londres. 1983.

9. Lorenz IN. Three Approaches to atmospheric predictability. Bull Am Meteorol Soc. 1969;50(5):345-351.

10. Scolaro LA, Roldan J, Theaux C, Damonte EB, Carlucci MJ. Experimental aspects suggesting a "fluxus" of information in the virions of herpes simplex virus populations. Front Microbiol. 2017;8:2625.

11. Theaux C. Analisis comparativo de la evolucion molecular del virus Herpes simplex 1. Tesis de Maestria en Biologia Molecular Medica, Universidad de Buenos Aires. Argentina. 2018.

12. Gleick J. Chaos. Making a new science. 1988.

13. Artuso MC, Roldán JS, Scolaro LA, Carlucci MJ. Viruses: as mediators in "elan vital" of the "creative" evolution. Infect Genet Evol. 2016;46:78-84.

14. Carlucci MJ, Damonte EB, Scolaro LA. Virus driven evolution: a probable explanation for "Similia Similibus Curantur" philosophy. Infect Genet Evol. 2011;11(5):798-802.

15. Cocchi F, Fusco D, Menotti L, Gianni T, Eisenberg RJ, Cohen $\mathrm{GH}$. The soluble ectodomain of herpes simplex virus $\mathrm{gD}$ contains a membrane-proximal pro-fusion domain and suffices to mediate virus entry. Proceedings of the National Academy of Sciences (PNAS) 2004;101(19):7445-7450.

16. Albiser G, Lamiri A, Premilat S. The A-B transition: temperature and base composition effects on hydration of DNA. Int J Biol Macromol. 2001;28(3), 199-203.

17. Arai S, Chatake T, Ohhara T, Kurihara K, Tanaka I, Suzuki N. Complicated water orientations in the minor groove of B-DNA decamer d(CCATTAATGG) 2 observed by neutron diffraction measurements. Nucleic Acids Res. 2005;33(9):3017-3024.

18. Chaplin M. Do we underestimate the importance of water in cell biology? Nat Rev Mol Cell Biol. 2006;7(11):861-866.

19. Furlong D, Swift H, Roizman B. Arrangement of herpes virus deoxyribonucleic acid in the core. J Virol. 1972;10(5):1071-1074.

20. Nazerian K. DNA configuration in the core of Mare' $k$ disease virus. J Virol. 1974;13(5):1148-1150.

21. Lipton B. The biology of belief. Unleashing the power of consciousness, matter and miracles. Chapter 3. Ed. Gaia Ediciones. Madrid. 2005.

22. Ruyechan WT, Morse LS, Knipe DM, Roizman B. Molecular genetics of herpes specifying the social behavior of infected cells. J Virol. 1979;29(2):677-697.

23. Roizman B, Sears AE. Herpes simplex viruses and their replication. Chapter 34. In Fundamental Virology. Second Edition, edited by Fields BN, Knipe DM et al. Raven Press, Ltd., New York. 1991.

24. Wheatley M. Leadership and the new science: learning about organization from an orderly universe. San Francisco, Berret-Koehler Publishers. 1992.

25. Capra F. The Web of Life: A new synthesis of mind and matter. London; Harper Collins Publishers. 1996.

26. Kaplan A. Development practitioners and social process. Artists of the Invisible. Ed. Pluto Press, London, England. 2002. 